\title{
Experiences and efficacy of noninvasive prenatal test using maternal plasma in single center: 1,591 cases
}

\author{
So Yeon Hong ${ }^{1 \oplus}$, So Hyun Shim ${ }^{2 \oplus}$, Hee Jin Park ${ }^{2 \oplus}$, Sung Shin Shim $^{2 \oplus}$, ji Youn Kim ${ }^{1 \oplus}$, Yeon Kyung $\mathrm{Cho}^{2 \oplus}$, Soo Hyun Kim ${ }^{2 \oplus}$ \\ and Dong Hyun $\mathrm{Cha}^{2}$ **i] \\ ${ }^{1}$ Department of Obstetrics and Gynecology, CHA Ilsan Medical Center, CHA University, Goyang, Korea \\ ${ }^{2}$ Department of Obstetrics and Gynecology, CHA Gangnam Medical Center, CHA University, Seoul, Korea
}

\begin{abstract}
Purpose: The objective of this study was to analyze the results of several noninvasive prenatal tests (NIPTs) from a single center and confirm their efficacy and reliability. In addition, we aimed to confirm the changes in the number of invasive tests performed after introducing NIPT.

Materials and Methods: NIPT data from a large single center from March 2014 to November 2018 were analyzed. Karyotyping was confirmed based on chorionic villus sampling, amniocentesis, or postnatal cord/peripheral blood sampling. Data on maternal age, gestational age, fetal fraction, and ultrasonographic results were analyzed. As the secondary outcome, the number of amniocentesis cases before and after the introduction of NIPT was compared. Results: Overall, 1,591 single pregnancy cases that underwent NIPT were enrolled. The mean maternal age was 36.05 (22-45) years. The average gestational age and fetal fraction were $12^{+1}\left(9^{+3}\right.$ to $\left.27^{+1}\right)$ weeks and $10.95 \%(3.6 \%$ to $31.3 \%)$, respectively. A total of 1,544 cases $(97.0 \%)$ were reported to have negative NIPT results and $40(2.5 \%)$ had positive NIPT results. The sensitivity and specificity of the overall abnormalities in NIPT were $96.29 \%$ and $99.36 \%$, respectively. The positive predictive value (PPV) and negative predictive value were $72.22 \%$ and $99.93 \%$ respectively. The mean number of amniocentesis cases were 21.7 per month (21.7 \pm 3.9$)$, which significantly decreased from 31.5 per month (31.5 \pm 4.8$)$ before conducting NIPT as a screening test. Conclusion: NIPT is currently a useful, powerful, and safe screening test. In particular, trisomy 21 is highly specific due to its high PPV. NIPT can reduce the potential risks of procedure-related miscarriages during invasive testing.
\end{abstract}

Key words: Cell-free nucleic acids, Noninvasive prenatal testing, Karyotype, Prenatal diagnosis.

\section{Introduction}

An important part of obstetric management is prenatal screening and diagnosis of chromosomal aberrations. In the past, many patients were managed by using combined nuchal translucency (NT) and biochemical first trimester screening [1].
Using these screening methods, the detection rate of aneuploidies was 95\% and the false positive rate was 3\% to 5\% [1]. To confirm the karyotyping, either first trimester chorionic villus sampling (CVS) or second trimester amniocentesis is required. These invasive procedures result in a miscarriage risk of $0.1 \%$ to $0.5 \%[2,3]$. However, the recent introduction of noninvasive

Received: 13 April 2020, Revised: 27 May 2020, Accepted: 27 May 2020, Published: 30 June 2020

${ }^{*}$ Corresponding author: Dong Hyun Cha, Ph.D. (D) https://orcid.org/0000-0003-0722-1714

Department of Obstetrics and Gynecology, CHA Gangnam Medical Center, 566 Nonhyeon-ro, Gangnam-gu, Seoul 06135, Korea.

Tel: +82-2-3468-3314, Fax: +82-2-558-1112, E-mail: chadh001@chamc.co.kr

Conflict of interest: The authors declare that they do not have any conflicts of interest.

(ㄷ) This is an open-access article distributed under the terms of the Creative Commons Attribution Non-Commercial License (http://creativecommons.org/licenses/by-nc/4.0/) which permits unrestricted non-commercial use, distribution, and reproduction in any medium, provided the original work is properly cited.

(c) Copyright 2020 by the Korean Society of Medical Genetics and Genomics 
prenatal tests (NIPT) that analyze DNA released from cytotrophoblastic cells into the maternal plasma has improved the detection of primary trisomies $(13,18$, and 21$)$ with a very low false-positive rate [4]. These tests also allow the detection of sex related chromosomal abnormalities [5]. NIPT has become less expensive over time, and many patients have chosen NIPT for screening.

The presence of fetal cell-free DNA (cfDNA) in the maternal circulation was first demonstrated by Lo et al. [6]. After its identification in 1997, a rapid increase in the use of cell-free fetal DNA analysis led to an awareness of the need for further development and implementation. There have been many studies into the use of cfDNA in maternal blood for the detection of trisomies 21, 18, and 13 in groups at high risk of aneuploidies $[4,7,8]$. A recent study showed excellent results when predicting aneuploidy in twin cases [9]. Another study reported an increase in the accuracy of NIPT, especially when performed at a late gestational stage [10]. For NIPT results, weighted pooled detection rates include $99.2 \%$ for trisomy $21,96.3 \%$ for trisomy 18 , and 91.0\% for trisomy 13. The concomitant false positive rates were 0.09\% for trisomy 21 and 0.13\% for trisomies 18 and 13 [11]. Significant amounts of data from large studies have been collected in other countries, but such data have not been collected for Korea. Therefore, it is important to analyze the results of NIPT in a large-scale Korean population to investigate its effectiveness in this population. The aim of this study was to collect data pertaining to NIPT experiences from clinical institutions, and assess the efficacy and reliability of the results in Korean women.

\section{Materials and Methods}

NIPT data were retrospectively collected from patients attending the CHA Gangnam Medical Center from March 2014 to November 2018. Patients who were referred from another hospital due to abnormal NIPT results were also included. All patients included in the study delivered a baby, and all samples were collected after the ninth week of gestation. Patients enrolled in this study included all of those who were examined using NIPT chromosomal aberration screening. There were cases in which patients only wanted to know the results if there were any abnormalities in the primary examination, or if there was an increased NT finding, or other abnormalities, on ultrasound examination.

Experienced maternal-fetal medicine specialists provided post-test counseling for all pregnant women who underwent NIPT. If the results were positive, either amniocentesis or CVS was suggested for karyotyping, as previously explained to the patients. Karyotyping confirmation was conducted by CVS in the first trimester and amniocentesis in the second trimester. The results were confirmed by postnatal cord blood sampling in cases where patients refused these invasive procedures. Details about maternal age, gestational age, body mass index (BMI), platform/ company, Z-score, fetal fraction, ultrasonographic results, indications for NIPT, and delivery results were analyzed. The monthly number of cases of amniocentesis was compared by randomizing the data from six months before and after the introduction of NIPT, and comparing them with the average number of cases.

Statistical analyses were performed using IBM SPSS ver. 20.0 (IBM Corp., Armonk, NY, USA). This study was approved by the institutional review board of the CHA Gangnam Medical Center, CHA University, Seoul, Korea (approval number: GCl-20-11).

\section{Results}

During the five-year review period, 12,265 women underwent either an integrated test or NIPT. Among these 12,265 women, $10,604(86.5 \%)$ chose the integrated test as their primary serum screening method and 1,661 (13.5\%) chose NIPT. Of the 1,661 NIPT patients, 1,591 were singleton pregnancies and 70 were twin pregnancies. Twin pregnancy cases were excluded from this study. A total of 1,591 patients who underwent NIPT in singleton pregnancies were therefore enrolled. Table 1 illustrates the clinical characteristics of the patients. The mean maternal age was 36.05 (range 22-45) years.

Most samples were tested in the first trimester or the beginning of the second trimester. The average gestational age was

Table 1. Clinical characteristics of the study population $(n=1,591)$

\begin{tabular}{lc}
\hline \multicolumn{1}{c}{ Characteristic } & Value \\
\hline Age $(\mathrm{yr})$ & $36.05(22-45)$ \\
Body mass index $\left(\mathrm{kg} / \mathrm{m}^{2}\right)$ & $21.5(15.8-41.3)$ \\
GA at sampling $(\mathrm{wk})$ & $12^{+1}\left(9^{+3}-27^{+1}\right)$ \\
Fetal fraction $(\%)$ & $10.95(3.6-31.3)$ \\
Indication of NIPT & \\
1st screening due to patient's desire & $1,391(87.4)$ \\
Age $<35 \mathrm{yr}$ & $367(26.4)$ \\
Age $\geq 35 \mathrm{yr}$ & $1,024(73.6)$ \\
Abnormal 1st screening result & $104(6.5)$ \\
Increased NT & $72(4.5)$ \\
Other abnormal ultrasound finding & $24(1.5)$ \\
\hline
\end{tabular}

Values are presented as mean (range) or number (\%).

GA, gestational age; NIPT, noninvasive prenatal test; NT, nuchal translucency. 
$12^{+1}\left(9^{+3}-27^{+1}\right)$ weeks. The mean maternal BMI was $21.5(15.8-$ 41.3) $\mathrm{kg} / \mathrm{m}^{2}$, and the average fetal fraction of reported samples was 10.95 (3.6-31.3)\%. When the average fetal fraction was derived by dividing by the $\mathrm{BMI}$, the average fetal fraction decreased as the BMI increased (Table 2). The indications for NIPT were as follows: first screening due to patient's request $(n=1,391$, 87.4\%); abnormal first screening results ( $n=104,6.5 \%)$; increased NT $(n=72,4.5 \%)$; and other abnormal sonographic findings $(n=24,1.5 \%)$.

Of the 1,591 patients, 1,544 (97.0\%) had negative NIPT results, and 40 cases (2.5\%) had positive NIPT results. Seven cases (0.4\%) were inconclusive $(\mathrm{FF}<4 \%)$ (Table 3). Of the 40 cases in the group with positive results, only 36 cytogenetic confirmation tests were performed and four cases were lost to follow up. The mean number of amniocenteses, (the secondary outcome) performed at our institution prior to the introduction of NIPT was 31.5 per month (31.5 \pm 4.8 ). However, this number decreased to $21.7 \mathrm{per}$ month after we began offering NIPT as a screening method (21.7 \pm 3.9$)$. The difference was statistically significant $(P<0.01)$.

The 36 confirmed cases produced the following results. Karyo-

Table 2. Mean fetal fraction by maternal BMI (GA11-12 weeks, $\mathrm{n}=1,287$ )

\begin{tabular}{lcc}
\hline BMI $\left(\mathrm{kg} / \mathrm{m}^{2}\right)$ & $\mathrm{n}$ & Mean fetal fraction (\%, range) \\
\hline$<18.5$ & 199 & $11.9(4.3-29.8)$ \\
$18.5-22.9$ & 797 & $11.4(4.0-26.5)$ \\
$23.0-24.9$ & 164 & $9.7(3.9-18.6)$ \\
$25.0-29.9$ & 102 & $8.18(4.3-29.8)$ \\
$\geq 30.0$ & 25 & $7.24(3.6-18.6)$ \\
\hline
\end{tabular}

BMI, body mass index; GA, gestational age. type tests in the NIPT trisomy 21 positive group revealed that all 19 cases were trisomy 21 (100.0\%). In two cases of trisomy 18, one case (50.0\%) was confirmed as trisomy 18. A single positive trisomy 13 was also confirmed as trisomy 13. The sex chromosome abnormality (SCA)-positive group of NIPT were XXX ( $n=3)$, $X X Y(n=3)$, and $X O(n=2)$, and four cases showed discordance, the result being a normal karyotype (three $46, X Y$ and one $46, X X$ ). Both trisomy 15 and trisomy 7 NIPT cases showed discordant results, and trisomy 14 was not confirmed.

Seven NIPT samples (0.4\%) were not analyzed due to low fetal fraction. Four patients returned for a repeat blood draw. However, the analysis failed a second time in three cases. Three patients underwent amniocentesis after the failed analysis. Of seven cases, six were confirmed by amniocentesis as having normal karyotypes. One pregnancy was terminated due to abnormal results-an exon 1-29 duplication of the dystrophin gene-after multiplex ligation-dependent probe amplification (MLPA) of the

Table 4. Seneitivity, specificity, PPV and NPV of NIPT in this study

\begin{tabular}{cccccc}
\hline & $\begin{array}{c}\text { Overall } \\
\text { abnormality }\end{array}$ & $\begin{array}{c}\text { Trisomy } \\
21\end{array}$ & $\begin{array}{c}\text { Trisomy } \\
18\end{array}$ & $\begin{array}{c}\text { Trisomy } \\
13\end{array}$ & SCA \\
\hline $\begin{array}{c}\text { Observed } \\
\text { sensitivity }\end{array}$ & $96.29 \%$ & $100 \%$ & N/A & N/A & N/A \\
$\begin{array}{c}\text { Observed } \\
\text { specificity }\end{array}$ & $99.36 \%$ & $100 \%$ & N/A & N/A & N/A \\
$\begin{array}{c}\text { Observed } \\
\text { PPV }\end{array}$ & $72.22 \%$ & $100 \%$ & N/A & N/A & N/A \\
$\begin{array}{c}\text { Observed } \\
\text { NPV }\end{array}$ & $99.93 \%$ & $100 \%$ & N/A & N/A & N/A \\
\hline
\end{tabular}

PPV, positive predictive value; NPV, negative predictive value; NIPT, noninvasive prenatal test; SCA, sex chromosome abnormality; N/A, not applicable.

Table 3. Results of NIPT analysis $(n=1,591)$

\begin{tabular}{|c|c|c|c|c|}
\hline & $\mathrm{n}$ & Confirmation test(+) & Concordance & Discordance \\
\hline \multicolumn{5}{|c|}{ Negative NIPT $(n=1,544,97.0 \%)^{a}$} \\
\hline \multicolumn{5}{|c|}{ Positive NIPT ( $n=40,2.5 \%)$} \\
\hline Trisomy 21 & 21 & 19 & 19 & 0 \\
\hline Trisomy 18 & 3 & 2 & 1 & 1 \\
\hline Trisomy 13 & 1 & 1 & 1 & 0 \\
\hline $\operatorname{SCA}(X, X X X, X X Y)$ & 8 & 8 & 4 & 4 \\
\hline Triploidy & 3 & 3 & 0 & 3 \\
\hline Trisomy 14 & 1 & 0 & & \\
\hline Trisomy 15 & 2 & 2 & 1 & 1 \\
\hline Trisomy 7 & 1 & 1 & 0 & 1 \\
\hline Total & 40 & 36 & 26 & 10 \\
\hline Uninformative results ( & \multicolumn{4}{|c|}{$\begin{array}{l}6 \text { cases were confirmed normal karyotype. } \\
1 \text { case was terminated of pregnancy. }\end{array}$} \\
\hline
\end{tabular}

NIPT, noninvasive prenatal test; SCA, sex chromosome abnormality.

${ }^{a}$ Discordance in 1 case (trisomy 18). ${ }^{b} 6$ cases were confirmed normal karyotype. 1 case was terminated of pregnancy. 
amniocentesis. All seven uninformative tests were conducted after 11 weeks gestation. Two patients were extremely obese $\left(\mathrm{BMl}>30 \mathrm{~kg} / \mathrm{m}^{2}\right)$. Even after a repeat NIPT, the results were "no call".

When we investigated the indications for NIPT, the proportion of chromosomal abnormalities in the NIPT positive group was the highest in patients with increased NT (>2.5 mm) (8.3\%). In other cases, the abnormality rate of the first positive screening was $6.7 \%$, and other abnormal ultrasound findings (4.1\%) were also observed.

The sensitivity and specificity of the NIPT were $96.29 \%$ and $99.36 \%$, respectively. In this study, the positive predictive value (PPV) was $72.22 \%$ and the negative predictive value (NPV) was 99.93\%. The PPV and NPV for each condition were calculated as shown in Table 4. The PPV and NPV for individual chromosome aberrations were not interpretable, with the exception of trisomy 21.

\section{Discussion}

Prenatal screening and diagnosis of chromosome aberrations are important aspects of prenatal care. A major objective in the field of prenatal testing is a reduction in the number of unnecessary invasive procedures. This is primarily achieved by the application of NIPT.

Women with a positive routine prenatal screening or an unusual ultrasound marker have a higher probability of selecting NIPT, as they generally want to avoid invasive procedures. Of the patients who underwent invasive tests, such as CVS or amniocentesis, 69\% were women with positive results from NIPT (data not shown). This observation indicates that the rate of NIPT prior to invasive testing has gradually increased. The number of amniocentesis cases significantly decreased in this institution following the implementation of NIPT. These results are similar to those of previous studies [12].

The groups that were tested because of abnormal findings such as an increased NT or an abnormal first screening had a higher percentage of positive results than NIPT results that were performed due to a patient's request. One reason for this finding may be that when women experienced an increase in their background risk, NIPT was seen as a noninvasive option for reducing risk. The most important benefit of NIPT, compared to conventional serum screening methods, is that the number of invasive diagnostic tests can be decreased.

However, it is important to overcome low fetal fraction, which can result in incomplete results for NIPT. In this study, seven samples could not be analyzed. Maternal obesity is the most well known cause of low fraction. In our cases, most of the low fractions were due to a low fetal fraction, and were associated with increased maternal weight. Successful analysis leading to reliable clinical results depends upon the percentage of fetal to maternal cfDNA in maternal plasma. The minimum cfDNA needed for analysis is reported to be approximately $5 \%$. In the present study we observed a correlation between fetal cfDNA fraction and maternal $\mathrm{BMI}$, as has been reported in previous studies. Currently, the American College of Obstetricians and Gynecologists (ACOG) recommends considering invasive diagnostic tests for those whose cfDNA fetal fraction is low in NIPT tests [13]. Unfortunately, other than gestational age and maternal weight, little is known about the clinical and biological factors influencing this parameter $[14,15]$. Therefore, additional research is required.

The diagnosis of trisomy 21 had an accuracy of 100\%, despite a fairly high number of cases. For other abnormalities, there were fewer patients to test. Thus, the NIPT remains at the limit of the screening tests. Rare fetal chromosome aneuploidies may involve all fetal autosomal chromosomal abnormalities other than the SCA and trisomies 13,18, and 21. However, most of the published data on NIPT have focused on three common aneuploidies: T13, T18, and T21; and SCAs. There have also been cases in which other minor aneuploidies (T15, T7, and T14) or gene mutations (exon 1-29 duplication in the dystrophin gene) have been diagnosed using NIPT. Further studies in this area are needed.

This study is valuable in that it provided data pertaining to a Korean population, which has previously been lacking. Strength of this work is that the positive NIPT results were supported by the results of invasive tests. The reliability of the NIPT could therefore be assessed in a single medical center.

One limitation of the study was that it was conducted by a single institution, and therefore included only a subset of the population. Due to the relatively small number of cases, it was difficult to determine the efficiency of the prenatal detection of fetal chromosomal abnormalities using NIPT. The number of chromosomal abnormalities detected was small, except for trisomy 21, resulting in a lack of data from which PPVs and sensitivities could be calculated. There was also a lack of age-specific PPVs. Two-thirds of the population were elderly and high-risk pregnant women, making it difficult to generalize the results to younger pregnant women.

Few studies have analyzed the results of NIPT in Korean women. One recent study analyzed two years of experience with NIPT [12]. The authors observed a significant decrease in the 
number of pregnant women who underwent invasive testing after NIPT was introduced. This reduction lowered the potential risks of procedure-related miscarriages associated with invasive testing [12]. The present study is of larger scale than the previous study. In addition, the previously reported sensitivity, specificity, and positive and negative predictions of NIPT were compared with current results. Not all of the women who received positive results from the NIPT choose to have a confirmation test, so the overall positive NIPT result ratio could be high.

It is important that this test be accompanied by genetic counseling. Patients must be educated about the limitations of the technology. This discussion should include the possibility of false positives, false negatives, and failed results that cannot be detected using current NIPT technology.

An increasing number of pregnant Korean women are choosing NIPT for screening. Further studies will be required to establish the error rates, accuracy, and reliability of its results.

In this study, a clinical review of the use of NIPT in general cases is introduced, based on results from a single institution. NIPT is a useful, powerful, and safe screening test, and can lead to a reduction in invasive testing. In particular, the diagnosis of trisomy 21 is highly specific, and has a high PPV. NIPT can be used to predict cases of trisomy 13 , trisomy 18 , and SCA, but a confirmation test is required. Our results indicate that NIPT is a valuable addition to standard prenatal screening, but further large-scale data analyses are required for the assessment of the value of NIPT to Korean women.

\section{References}

1. Nicolaides KH. Screening for fetal aneuploidies at 11 to 13 weeks. Prenat Diagn 2011;31:7-15.

2. Simpson JL. Invasive procedures for prenatal diagnosis: any future left? Best Pract Res Clin Obstet Gynaecol 2012;26:625-38.

3. Akolekar R, Beta J, Picciarelli G, Ogilvie C, D'Antonio F. Procedurerelated risk of miscarriage following amniocentesis and chorionic villus sampling: a systematic review and meta-analysis. Ultrasound Obstet Gynecol 2015;45:16-26.

4. Benn P, Cuckle $H$, Pergament E. Non-invasive prenatal testing for aneuploidy: current status and future prospects. Ultrasound Obstet
Gynecol 2013;42:15-33.

5. Ramdaney A, Hoskovec J, Harkenrider J, Soto E, Murphy L. Clinical experience with sex chromosome aneuploidies detected by noninvasive prenatal testing (NIPT): accuracy and patient decision-making. Prenat Diagn 2018;38:841-8.

6. Lo YM, Corbetta N, Chamberlain PF, Rai V, Sargent IL, Redman CW, et al. Presence of fetal DNA in maternal plasma and serum. Lancet 1997;350:485-7.

7. Iwarsson $E_{1}$ Jacobsson $B$, Dagerhamn J, Davidson $T$, Bernabé $E_{1}$ Heibert Arnlind M. Analysis of cell-free fetal DNA in maternal blood for detection of trisomy 21, 18 and 13 in a general pregnant population and in a high risk population - a systematic review and metaanalysis. Acta Obstet Gynecol Scand 2017;96:7-18.

8. Flöck A, Tu NC, Rüland A, Holzgreve W, Gembruch U, Geipel A. Noninvasive prenatal testing (NIPT): Europe's first multicenter post-market clinical follow-up study validating the quality in clinical routine. Arch Gynecol Obstet 2017;296:923-8.

9. Fosler $L$, Winters $P$, Jones KW, Curnow KJ, Sehnert AJ, Bhatt $S$, et al. Aneuploidy screening by non-invasive prenatal testing in twin pregnancy. Ultrasound Obstet Gynecol 2017;49:470-7.

10. Taneja PA, Prosen TL, de Feo E, Kruglyak KM, Halks-Miller M, Curnow $\mathrm{K}$, et al. Fetal aneuploidy screening with cell-free DNA in late gestation. J Matern Fetal Neonatal Med 2017;30:338-42.

11. Gil MM, Accurti V, Santacruz B, Plana MN, Nicolaides KH. Analysis of cell-free DNA in maternal blood in screening for aneuploidies: updated meta-analysis. Ultrasound Obstet Gynecol 2017;50:302-14.

12. Noh JJ, Ryu HM, Oh SY, Choi SJ, Roh CR, Kim JH. A two-year experience of non-invasive prenatal testing (NIPT) at an urban tertiary medical center in South Korea. Taiwan J Obstet Gynecol 2019;58:54551.

13. ACOG Committee on Obstetric Practice. ACOG Committee opinion no. 475: antenatal corticosteroid therapy for fetal maturation. Obstet Gynecol 2011;117(2 Pt 1):422-4.

14. Canick JA, Palomaki GE, Kloza EM, Lambert-Messerlian GM, Haddow JE. The impact of maternal plasma DNA fetal fraction on next generation sequencing tests for common fetal aneuploidies. Prenat Diagn 2013;33:667-74.

15. Wang E, Batey A, Struble C, Musci T, Song K, Oliphant A. Gestational age and maternal weight effects on fetal cell-free DNA in maternal plasma. Prenat Diagn 2013;33:662-6. 